Received: $\quad 2016.02 .05$

Accepted: 2016.03.11

Published: 2016.04.21

\title{
Hyperamylasemia as an Early Predictor of Mortality in Patients with Acute Paraquat Poisoning
}

Authors' Contribution: Study Design A Data Collection B Statistical Analysis C Data Interpretation D Manuscript Preparation E Literature Search F Funds Collection G
ABCDEF 1 Shuai Liu*

ABCDEF 1 Qiang Wang*

$A B C D 2$ Rong Zhou

BCD 1 Changbin Li

$B C D$ Dayong Hu

BCD 1 Wen Xue

E 2 Tianfu Wu

E 1,3 Chandra Mohan

acdefg 1 Ai Peng
1 Department of Nephrology \& Rheumatology, Shanghai Tenth People's Hospital, Tongi University School of Medicine, Shanghai, P.R. China

2 Department of Nephrology, Shanghai Yangpu Hospital, Tongji University School of Medicine, Shanghai, P.R. China

3 Department of Biomedical Engineering and Pharmacy, University of Houston, Houston, TX, U.S.A.
Corresponding Author: Source of support:

* These authors contributed equally to this work

Ai Peng, e-mail: pengai@tongji.edu.cn

This work was supported by grants from the National Natural Science Foundation of China $(81171792,81270136)$ and the "Thousand Foreign Talents Program of China" (WQ20133100165)

Background:

Material/Methods:

Results:

Conclusions:

\section{MeSH Keywords:}

Full-text PDF:
Paraquat (PQ) is a non-selective and fast-acting contact herbicide which has been widely used in developing countries. Hyperamylasemia was reported in patients with $\mathrm{PQ}$ poisoning. This study investigated the predictive value and clinical characteristics of hyperamylasemia in patients with $\mathrm{PQ}$ poisoning.

This study included 87 patients with acute PQ poisoning admitted from July 2012 to May 2015. Data were collected from medical records. Receiver operating characteristic (ROC) analysis was conducted to analyze the discriminatory potential of serum amylase with respect to 90-day mortality.

Of 87 patients, 29 patients had elevated serum amylase. We found that serum amylase was significantly higher among patients with AKI than those with non-AKI $(p<0.001)$, and was an independent predictor of mortality (hazard ratio $[\mathrm{HR}]=3.644 ; 95 \%[\mathrm{Cl}], 1.684-7.881 ; p=0.001$ ). The area under the ROC curve for the serum amylase (area under curve $[\mathrm{AUC}]=0.796 ; 95 \%[\mathrm{Cl}], 0.690-0.903$ ) had a better discriminatory potential than plasma $\mathrm{PQ}$ concentration $(0.698 ; 0.570-0.825)$ or urinary $\mathrm{PQ}$ concentration $(0.647 ; 0.514-0.781)$ in predicting 90 -day mortality.

Hyperamylasemia is a valuable early predictor of 90-day mortality in PQ poisoning.

Hyperamylasemia $\bullet$ Mortality $\bullet$ Paraquat

http://www.medscimonit.com/abstract/index/idArt/897930 


\section{Background}

Paraquat (PQ) is a non-selective and fast-acting contact herbicide which has been widely used in developing countries [1,2]. However, $\mathrm{PQ}$ is highly toxic to humans and animals. $\mathrm{PQ}$ poisoning has become a clinical challenge due to the lack of specific antidote or effective treatment [3]. In recent decades, there have been numerous fatalities caused by accidental or voluntary ingestion of $P Q[4,5]$.

A variety of parameters has been suggested to predict clinical outcome after ingestion of $\mathrm{PQ}$, including plasma and urine $\mathrm{PQ}$ concentrations, urinary PQ qualitative test, liver enzymes, serum creatinine, arterial blood bicarbonate, leukocytosis, angiopoietin, and acidosis [6-14]. In addition, severity scoring systems such as sequential organ failure assessment (SOFA), acute physiology and chronic health evaluation II (APACHE II), simplified acute physiology score II (SAPS II), and severity index for paraquat poisoning (SIPP) have been proposed as disease progression predictors [15-19]. The extent of lung injury in CT and pulmonary emphysema have also been claimed to correlate with the prognosis of PQ poisoning [20,21]. Although much effort has been expended in exploring effective predictors, most of those potential predictors have not been independently validated, and some of the indicators have proven to be too complex or unfavorable for early prediction $[10,12]$. Therefore, further efforts are warranted to develop reliable predictors for prognosis in PQ poisoning and to guide future clinical interventions.

The main sources of amylase are the pancreas and salivary glands. Serum amylase is typically used as confirm or exclude the diagnosis of pancreatitis and salivary glands disease. However, increase in serum level of amylase frequently occurs in other conditions, such as in patients with shock, trauma, hypoxemia, kidney damage, and acute liver failure [22-24]. Hyperamylasemia was also reported to occur in patients with PQ poisoning $[10,25,26]$. Here, we report the predictive value and clinical characteristics of hyperamylasemia in patients with $\mathrm{PQ}$ poisoning.

\section{Material and Methods}

The protocol of this study was approved by the Shanghai Tenth People's Hospital of Tongji University Institutional Review Board (IRB: 2012RES045).

\section{Patients}

We reviewed the medical records of patients admitted to Shanghai Tenth People's Hospital with acute PQ poisoning from July 2012 to May 2015. During this period, 102 patients with

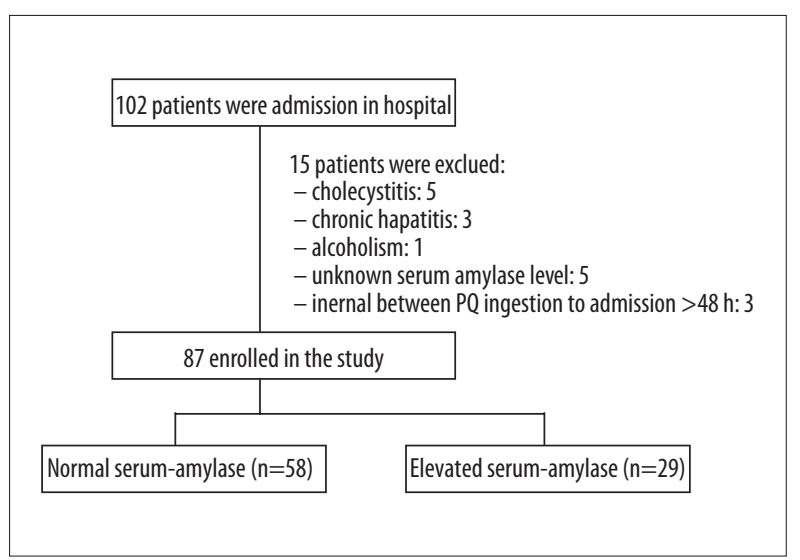

Figure 1. Flow chart showing enrollment of patients.

PQ poisoning were admitted to this hospital; 87 patients were included in our research eventually according to the inclusion criteria and exclusion criteria (Figure 1). Inclusion criteria were: (1) the patient's age between 15 to 65 years old, and (2) plasma or urine PQ concentration exceeding $0.1 \mathrm{mg} / \mathrm{L}$. Exclusion criteria were: (1) previous diagnosis of the following diseases: patients with history of pancreatic disease, renal function impairment, symptomatic biliary stones, or chronic hepatitis; (2) patients with a history of alcoholism; (3) patients without serum amylase measurement; and (4) interval between PQ ingestion and hospital admission $>48 \mathrm{~h}$.

\section{Data collection}

Demographic data, medical histories, nausea and vomiting, stomach ache, quantity of $P Q$ ingested, time from $P Q$ poisoning to treatment, and APACHE II and SOFA scores were collected. APACHE II and SOFA scores were based on the worst condition within the first $24 \mathrm{~h}$ of admission. Clinical laboratory tests completed during $24 \mathrm{~h}$ of hospitalization were collected, including measurements of serum amylase, plasma and urine $\mathrm{PQ}$ concentrations, $\mathrm{CRP}$, glucose, $\mathrm{Ca}^{2+}$, routine blood test, blood gas analysis, serum creatinine ( $\mathrm{SCr}$ ), blood urea nitrogen (BUN), aspartate aminotransferase (AST), and alanine aminotransferase (ALT). Abdominal CT scan reports were recorded when available. The primary outcome measure was the occurrence of death within 90 days after $P Q$ ingestion.

\section{Treatment protocol}

All patients received standard of care treatment, as described in our previous study $[20,21]$. In brief, all subjects admitted to the Emergency Department were treated with gastric lavage and activated charcoal. After that, charcoal hemoperfusion or continuous renal replacement therapy (CRRT) was performed if needed. Other treatment modalities such as methylprednisolone pulse treatment, prevention and treatment of infection, and protection of vital organs were implemented as clinically warranted. 


\section{Definition}

Toxic hepatitis was defined as having either AST or ALT >80 IU/L (twice the upper normal limits) or a total bilirubin level $>36.6$ umol/L [27]. Hypoxemia was defined as $\mathrm{PaO} 2<70 \mathrm{mmHg}$ [27]. Acute kidney injury (AKI) was diagnosed based on the KDIGO criteria (increase in serum creatinine by $\geq 0.3 \mathrm{mg} / \mathrm{dl}(\geq 26.5 \mu \mathrm{mol} / \mathrm{l}$ ) within $48 \mathrm{~h}$ or an increase in serum creatinine to $\geq 1.5$ times baseline within the preceding 7 days) [28]. Hyperamylasemia was defined as having serum amylase levels greater than the normal levels ( $>220 \mathrm{U} / \mathrm{L})$. Diagnosis of acute pancreatitis requires 2 of the following 3 features: (1) upper abdominal pain of acute onset often radiating through to the back, (2) serum amylase or lipase activity greater than 3 times normal, and (3) positive abdominal CT results $[29,30]$.

\section{Statistical analysis}

Univariate analysis was performed with nonparametric test to evaluate differences in continuous variables and the chi-square test was used for comparing distributions among groups. To estimate hazard ratios and the resulting $95 \%$ confidence intervals (Cls) of predictors of in-hospital mortality, a forward stepwise Cox proportional hazards model was used. Survival curves were estimated using the Kaplan-Meier method and were compared using the log-rank test. Receiver operating characteristic (ROC) analysis was used to study the discriminatory power of serum amylase with respect to 90 -day mortality. All tests were 2 -tailed; $p$ values $<0.05$ were considered to indicate statistical significance. Data were analyzed using SPSS version 20.0 for Microsoft Windows (SPSS, Inc., Chicago, IL, USA).

\section{Results}

\section{Patient characteristics}

A total of 87 patients with PQ poisoning (36 men and 51 women) were included in this study. Overall 90-day mortality was $41.4 \%$ (36 of 87 ). We divided patients into 2 groups in accordance with serum amylase levels ("non-elevation group" and "elevation group"); 29 Patients had elevated serum amylase in this study (Figure 1). Univariate analysis identified that time to hospital after ingestion time $(p=0.020)$, estimated PQ amount $(p<0.001)$, APACHE II $(p<0.001)$ and SOFA $(p<0.001)$ scores, and mortality rate $(p=0.001)$ were significantly higher in patients with hyperamylasemia (in the elevation group in Table 1). In contrast, there were no significant differences in age and sex between the non-elevation group and elevation group. Stomach ache was also found to be similar in the 2 groups.

\section{Association between serum amylase and laboratory parameters of $P Q$ poisoning}

Next, we compared the laboratory parameters between the serum amylase elevation group and serum amylase non-elevation group. Plasma $\mathrm{PQ}$ concentration $(p=0.001)$, urine $\mathrm{PQ}$ concentration ( $p=0.001), \mathrm{CRP}(p<0.001), \mathrm{WBC}(p<0.001), \mathrm{SCr}$ $(p<0.001)$, AST $(p<0.001)$, ALT $(p<0.001)$, and PaCO2 $(p=0.001)$ were significantly increased in patients with hyperamylasemia. In addition to the above variables, other laboratory parameters such as glucose and $\mathrm{Ca}^{2+}$ did not show any significant difference (Table 1).

\section{Risk factor analysis for 90-day mortality}

In the univariate Cox proportional hazard regression model, we used age, sex, time to hospital, estimated PQ amount, serum amylase, SOFA scores, APACHE II scores, and plasma and urine $\mathrm{PQ}$ concentrations as independent variable. This analysis confirmed that the estimated $\mathrm{PQ}$ amount $(p<0.001)$, serum amylase $(p<0.001)$, SOFA scores $(p<0.001)$, APACHE II scores $(p<0.001)$, and plasma $(p<0.001)$ and urine $(p<0.001) \mathrm{PQ}$ concentrations were all significantly associated with 90-day mortality. Next, a forward stepwise multiple Cox proportional hazards model indicated that the probability of mortality increased with serum amylase (hazard ratio $[\mathrm{HR}]=3.644 ; 95 \%[\mathrm{Cl}], 1.684-7.881$; $p=0.001)$, APACHE II scores $(3.518 ; 1.310-9.446 ; 0.013)$ and plasma PQ concentration $(4.296 ; 2.164-8.528 ; 0.004)$ (Table 2). Table 2 indicates that serum amylase was independently associated with an increased risk of death. A log-rank test further confirmed that hyperamylasemia was associated with higher 90-day mortality (95\% [Cl], 14.100-43.495; p<0.001) (Figure 2).

\section{ROC analysis}

We calculated the areas under ROC curve of plasma PQ concentration, urinary $\mathrm{PQ}$ concentration, serum amylase, serum creatinine, and APACHE II and SOFA scores to compare the discriminatory capacities of these parameters in predicting 90 day mortality. The area under the ROC curve for serum amylase (area under curve $[A U C]=0.837 ; 95 \%$ [Cl], 0.750-0.923) exhibited better discriminatory potential than plasma PQ concentration $(0.679 ; 0.562-0.796)$ or urinary PQ concentration (0.647; 0.528-0.766), although it had predictive potential similar to APACHE II $(0.845 ; 0.765-0.925)$ and SOFA $(0.867 ; 0.790$ 0.943 ) scores (Figure 3).

\section{Association between serum amylase and organ damage in PQ poisoning}

Among the patients with hyperamylasemia, 25 of 29 (86.2\%) patients developed AKI ( $p<0.001)$, while 20 of $29(69.0 \%)$ patients exhibited hepatotoxicity $(p=0.000)$ (Table 1$)$. Indeed, the 
Table 1. General characteristics and laboratory data within $24 \mathrm{~h}$ following admission.

\begin{tabular}{|c|c|c|c|c|c|}
\hline Characteristic & \multicolumn{2}{|c|}{ Non-elevation group $(n=58)$} & \multicolumn{2}{|c|}{ Elevation group $(n=29)$} & P value \\
\hline Age, year & \multicolumn{2}{|r|}{$34.4 \pm 2.6$} & \multicolumn{2}{|r|}{$37.8 \pm 4.6$} & 0.643 \\
\hline Male/Female, $n$ & \multicolumn{2}{|r|}{$28 / 30$} & \multicolumn{2}{|r|}{$8 / 21$} & 0.065 \\
\hline Time to hospital after ingestion, hour & 24 & $(1-267)$ & 13 & $(6-108)$ & 0.020 \\
\hline Estimated $\mathrm{PQ}$ amount, $\mathrm{ml}$ & 15 & $(2-120)$ & 60 & $(10-200)$ & 0.000 \\
\hline Hemoperfusion, $\mathrm{n}$ & 39 & $(67)$ & 23 & (79) & 0.241 \\
\hline Nausea and vomiting, n (\%) & 13 & $(22)$ & 15 & $(52)$ & 0.006 \\
\hline Stomachache, n (\%) & 11 & (19) & 6 & $(21)$ & 0.848 \\
\hline SOFA & 2 & $(0-9)$ & 6 & $(1-12)$ & 0.000 \\
\hline APACHE II & 2 & $(0-14)$ & 9 & $(1-24)$ & 0.000 \\
\hline Nonsurvivors, n (\%) & 12 & $(21)$ & 24 & $(83)$ & 0.000 \\
\hline Plasma PQ concentration, mg/L & 0.01 & $(0.01-22.28)$ & 0.49 & $(0.01-408.10)$ & 0.001 \\
\hline Urine $\mathrm{PQ}$ concentration, $\mathrm{mg} / \mathrm{L}$ & 2.08 & $(0.01-248.72)$ & 35.58 & $(0.01-2762.10)$ & 0.001 \\
\hline CRP & 3.5 & $(0.4-68.0)$ & 19.7 & $(0.5-126.0)$ & 0.000 \\
\hline Glucose & 6.1 & $(2.1-21.6)$ & 7.6 & $(2.9-17.4)$ & 0.054 \\
\hline $\mathrm{Ca}^{2+}$ & 2.3 & $(1.8-3.0)$ & 2.3 & $(1.4-3.0)$ & 0.939 \\
\hline \multicolumn{6}{|l|}{ Routine blood test } \\
\hline WBC & 10.2 & $(4.2-29.3)$ & 22.5 & $(10.6-46.4)$ & 0.000 \\
\hline $\mathrm{Hb}$ & 128.0 & $(87.0-177.0)$ & 4.0 & $(1.0-17.2)$ & 0.811 \\
\hline PLT & 145.5 & $(40.0-363.0)$ & 156.0 & $(33.0-306.0)$ & 0.322 \\
\hline \multicolumn{6}{|l|}{ Renal function } \\
\hline AKI, n (\%) & 27 & $(46.6)$ & 25 & $(86.2)$ & 0.000 \\
\hline BUN, $\mathrm{mmol} / \mathrm{L}$ & 5.4 & $(0.5-55.9)$ & 7.0 & $(1.9-40.3)$ & 0.089 \\
\hline $\mathrm{SCr}, \mathrm{umol} / \mathrm{L}$ & 93.8 & $(29.6-999.8)$ & 172.0 & $(37.1-821,9)$ & 0.000 \\
\hline \multicolumn{6}{|l|}{ Liver function } \\
\hline Toxic hepatitis, n (\%) & 5 & $(8.6)$ & 20 & $(69.0)$ & 0.000 \\
\hline Serum AST, IU/L & 25.8 & $(7.9-328.5)$ & 190.8 & $(12.0-705.1)$ & 0.000 \\
\hline Serum ALT, IU/L & 18.6 & $(4.9-562.1)$ & 162.5 & $(6.0-1327.6)$ & 0.000 \\
\hline \multicolumn{6}{|l|}{ Arterial blood gases } \\
\hline Hypoxemia, n (\%) & 8 & $(13.8)$ & 8 & $(27.6)$ & 0.146 \\
\hline Acidosis, n (\%) & 4 & (6.9) & 6 & $(20.7)$ & 0.077 \\
\hline $\mathrm{PH}$ & 7.42 & $(7.20-7.50)$ & 7.40 & $(7.21-7.54)$ & 0.380 \\
\hline $\mathrm{PaO}_{2}, \mathrm{mmHg}$ & 90.0 & $(37.0-131.0)$ & 88.2 & $(30.0-187.0)$ & 0.989 \\
\hline $\mathrm{PaCO}_{2}, \mathrm{mmHg}$ & 37.7 & $(21.0-51.0)$ & 31.0 & $(0.0-45.0)$ & 0.001 \\
\hline
\end{tabular}

PQ - paraquat; APACHE - Acute Physiology and Chronic Health Evaluation; SOFA - Sequential Organ Failure Assessment; CRP C-reactive protein; WBC - white blood cell; Hb - hemoglobin; PLT - platelet; AKI - acute kidney injury; BUN - blood urea nitrogen; SCr - serum creatinine; AST - aspartate aminotransferase; ALT - alanine aminotransferase. Data are presented as means \pm SD or median (interquartile range) and categorical variable is presented as no (\%). 
Table 2. Cox proportional hazards models for mortality prediction in $\mathrm{PQ}$ poisoning.

\begin{tabular}{|c|c|c|c|c|c|c|}
\hline & \multicolumn{3}{|c|}{ Univariate COX Model } & \multicolumn{3}{|c|}{ Multivariate COX Model } \\
\hline & \multicolumn{2}{|c|}{$\mathrm{HR}(95 \% \mathrm{Cl})$} & \multirow{2}{*}{$\begin{array}{l}\text { P value } \\
0.959\end{array}$} & \multirow{2}{*}{\multicolumn{2}{|c|}{$\begin{array}{c}\text { HR }(95 \% \mathrm{Cl}) \\
\text { N/A }\end{array}$}} & \multirow{2}{*}{$\begin{array}{c}\text { P value } \\
\text { N/A }\end{array}$} \\
\hline Age & 1.000 & $(0.985-1.015)$ & & & & \\
\hline Gender & 0.974 & $(0.502-1.891)$ & 0.849 & & N/A & N/A \\
\hline Time to hospital & 0.999 & $(0.992-1.006)$ & 0.849 & & N/A & N/A \\
\hline Estimated PQ amount & 7.685 & $(3.343-17.667)$ & 0.000 & & N/A & 0.171 \\
\hline Serum amylase & 8.823 & (4.336-17.954) & 0.000 & 3.644 & $(1.684-7.881)$ & 0.001 \\
\hline SOFA & 3.480 & $(1.754-6.904)$ & 0.000 & 3.465 & $(0.962-12.474)$ & 0.057 \\
\hline APACHE II & 3.639 & $(1.876-7.059)$ & 0.000 & 3.518 & $(1.310-9.446)$ & 0.013 \\
\hline Plasma PQ concentration & 4.296 & $(2.164-8.528)$ & 0.000 & 2.714 & $(1.373-5.364)$ & 0.004 \\
\hline Urine $\mathrm{PQ}$ concentration & 2.566 & $(1.278-5.153)$ & 0.000 & & N/A & 0.546 \\
\hline
\end{tabular}

HR - hazard ratio; N/A - not applicable; SOFA - Sequential Organ Failure Assessment; APACHE II - Acute Physiology and Chronic Health Evaluation.

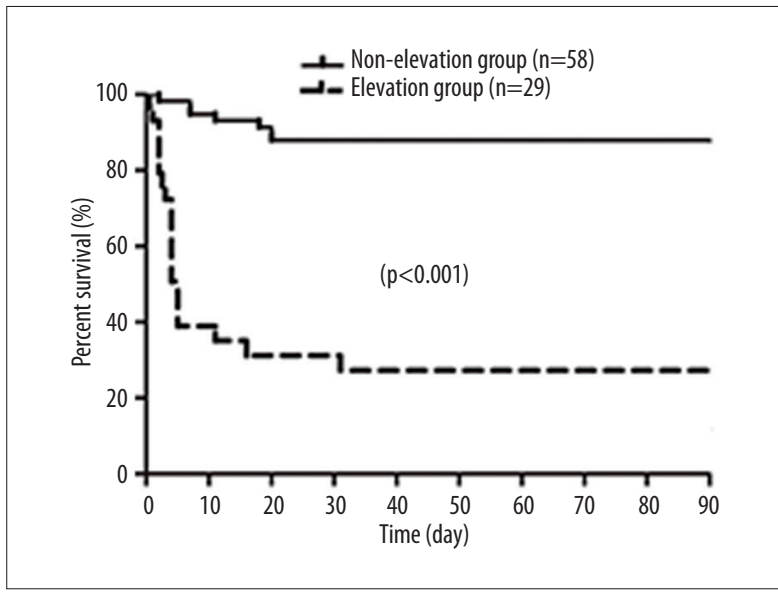

Figure 2. Kaplan-Meier survival curves of 87 paraquat poisoning cases stratified according to serum amylase. The $p$ values were derived using a log-rank test.

serum amylase levels were significantly higher among patients with AKI compared to those without AKI (Figure 4). Hypoxemia and acidosis showed no significant difference between the 2 groups (Table 1 ). Only 1 patient (3.4\%) was diagnosed with $\mathrm{PQ}$-associated painless acute pancreatitis (data not shown).

\section{Discussion}

In the present study, univariate analysis indicated that patients with hyperamylasemia exhibited higher plasma level of $P Q$, urinary level of $P Q$, estimated PQ amount, and APACHE II and SOFA scores. Hyperamylasemia was also correlated with mortality and the severity of AKI and toxic hepatitis (Table 1).

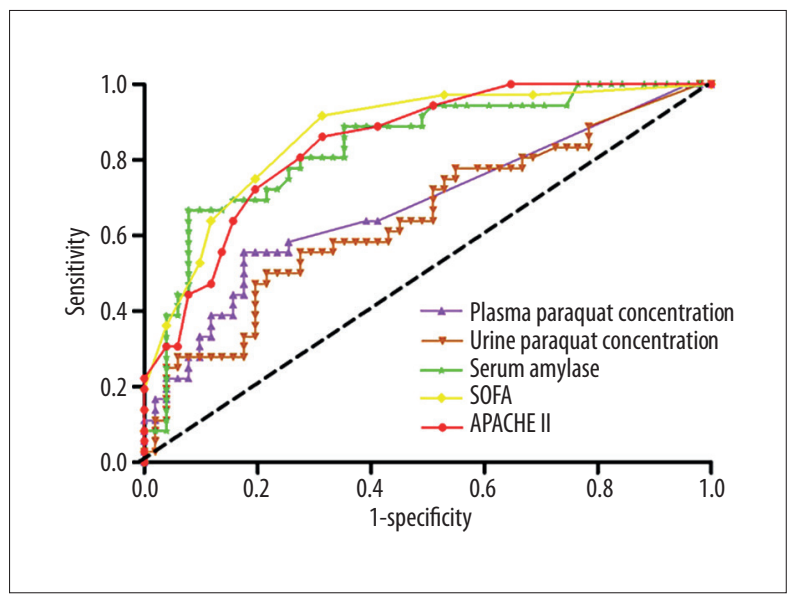

Figure 3. The receiver operating characteristic (ROC) curves constructed for 90-day mortality outcome prediction using scores of Acute Physiology and Chronic Health Evaluation (APACHE) II and Sequential Organ Failure Assessment (SOFA), serum amylase, plasma paraquat concentration, and urine paraquat concentration in $\mathrm{PQ}$ poisoning.

Our study agrees well with previous studies that elevated amylase activity is a significant predictor of survival in $\mathrm{PQ}$ poisoning $[10,25,31]$. These findings suggest that hyperamylasemia could be used as a valuable and objective predictive parameter for mortality in $\mathrm{PQ}$ poisoning.

Li et al. found that amylase is an independent prognostic marker, and the ROC curve showed serum amylase has a good power in the prediction of the death [26]. Our study was consistence with theirs (Table 2). However, in the present study we 


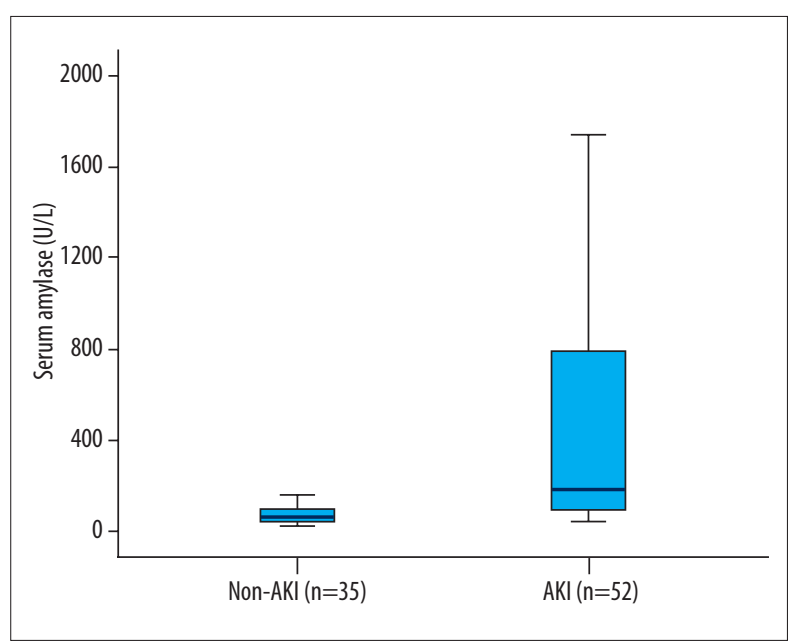

Figure 4. Boxplot showing minimum, maximum, median, and $5^{\text {th }}$ and $95^{\text {th }}$ percentiles of serum amylase in patients without acute kidney injure (non-AKI) and acute kidney injure (AKI).

further compared the predictive power of amylase with plasma and urine concentrations of paraquat, and found that the discriminatory power of serum amylase in our study was much better than plasma and urine PQ concentration (Figure 3).

Plasma and urine concentrations have been regarded as effective parameters for prediction of clinical outcome following acute $\mathrm{PQ}$ poisoning $[2,7,8]$. However, these have been questioned, as some studies found that plasma concentration was not as reliable as reported [32,33]. The mortality predictive powers of plasma and urine $\mathrm{PQ}$ concentrations were relatively low in our study. This may be explained by the following: (1) serum paraquat distributes to other organs quickly and decreases rapidly within the first few hours after ingestion. During this period, the plasma PQ concentration varies substantially even with slight changes in the time interval following ingestion [32]. (2) In the present study, the time interval between ingestion and serum $\mathrm{PQ}$ measurement was quite variable between the patients; thus, the varying or increased time-to-hospital interval after PQ ingestion may account for the observation that plasma $P Q$ concentrations are not predictive of outcome. (3) PQ is primarily eliminated through the kidneys, and renal excretion of $P Q$ is decreased when renal function is compromised [32]. Indeed, AKI occurred in 52 of 87 patients (59.8\%), and this may have influenced the predictive capacity of urine PQ concentrations. (4) Charcoal hemoperfusion was initiated if the urine dithionite test was positive in $\mathrm{PQ}$-poisoned patients. Thus, the initial management with charcoal hemoperfusion in $\mathrm{PQ}$ poisoning may also account for the observation that blood and urine PQ concentrations were not predictive of outcome in the present study.
In our study, 29 of 87 patients (33.3\%) exhibited hyperamylasemia and more than half of them exhibited 3-fold or higher increases. Of these, 1 of 29 (3.4\%) patients were diagnosed with PQ-associated painless mild acute pancreatitis and eventually died. A previous study on $\mathrm{PQ}$ poisoning reported that mild acute pancreatitis occurred in 1 out of $8 \mathrm{PQ}$-poisoned autopsy cases [34]. It is possible that hyperamylasemia may reflect clinical acute pancreatitis in a small number of cases. Taking into account that elevations of non-pancreatic amylase have been reported in a large number of pathologies, there may have been some other causes of elevated serum amylase in PQ-poisoned patients.

Some earlier studies suggested that the reason for increased serum levels of serum amylase may be due to a decreased rate of excretion into the urine, rather than direct pancreatic cellular damage [35], such as in cardiovascular surgery [36]. It has been reported that about $25 \%$ of serum amylase is excreted by the kidneys [37]. Kidney injury can decrease the excretion of serum amylase and this has been associated with elevation of pancreatic isoamylase [38,39]. Unfortunately, early deterioration of renal function is a critical complication in acute $P Q$ poisoning [40,41]. There were 25 of the 29 patients (86.2\%) with hyperamylasemia combined with AKI in our study. Serum amylase was significantly higher among patients with AKI than those without AKI (Figure 4). These findings raise the possibility that AKI may be an important cause of elevated serum amylase in PQ-poisoned patients.

Pancreatic damage secondary to acute hepatic failure has been described in many reports [42-44]. Toxic hepatitis occurred in 25 of 87 patients $(28.7 \%)$ in our study; only 1 of them progressed to acute hepatic failure and showed no evidence of hyperamylasemia. Hence, toxic hepatitis was not a common cause for the hyperamylasemia observed in our study. Other potential causes of elevated amylase, such as hypoxemia, have been observed in other studies [24]. However, hypoxemia was not different among PQ-poisoned patients with hyperamylasemia compared to those with normal values (Table 1), indicating that hypoxemia was not causing the hyperamylasemia observed in PQ-poisoned patients.

The hyperamylasemia in $\mathrm{PQ}$ poisoning also may also originate from cell injury in other organs, such as salivary glands and the intestines. In addition, although only a minority of PQ-poisoned patients were diagnosed with clinically acute pancreatitis, PQinduced subclinical pancreatic damage could not be excluded. Pancreatic amylase (P-Amy) and the salivary amylase(S-Amy) are 2 specific amylase isoenzymes [45]. P-Amy has a better sensitivity and specificity than total serum amylase for diagnosing acute pancreatitis [46], which will be monitored in this laboratory in the future. 


\section{Conclusions}

Serum amylase is frequently elevated in PQ-poisoned patients, although its mechanism remains to be established. Hyperamylasemia is an early and valuable predictor for 90-day mortality in $\mathrm{PQ}$ poisoning, and this is a simpler and more objective test compared to the APACHE II or SOFA scores.

\section{References:}

1. Hart TB: Paraquat - a review of safety in agricultural and horticultural use. Hum Toxicol, 1987; 6: 13-18

2. Senarathna L, Eddleston M, Wilks MF et al: Prediction of outcome after paraquat poisoning by measurement of the plasma paraquat concentration. QJM, 2009; 102: 251-59

3. Sittipunt C: Paraquat poisoning. Respir Care, 2005; 50: 383-85

4. Eddleston $M$ : Patterns and problems of deliberate self-poisoning in the developing world. QJM, 2000; 93: 715-31

5. Olson DP, Diaz JA, Jereda JD: A fatal case of paraquat ingestion: Clinical course and review of pathophysiology. Med Sci Monit, 2010; 16(12): CS153-56

6. Hart TB, Nevitt A, Whitehead A: A new statistical approach to the prognostic significance of plasma paraquat concentrations. Lancet, 1984; 2: 1222-23

7. Scherrmann JM, Houze P, Bismuth C, Bourdon R: Prognostic value of plasma and urine paraquat concentration. Hum Toxicol, 1987; 6: 91-93

8. Proudfoot AT, Stewart MS, Levitt T, Widdop B: Paraquat poisoning: Significance of plasma-paraquat concentrations. Lancet, 1979; 2: 330-32

9. Jones AL, Elton R, Flanagan R: Multiple logistic regression analysis of plasma paraquat concentrations as a predictor of outcome in 375 cases of paraquat poisoning. QJM, 1999; 92: 573-78

10. Lee EY, Hwang KY, Yang JO, Hong SY: Predictors of survival after acute paraquat poisoning. Toxicol Ind Health, 2002; 18: 201-6

11. Hong SY, Yang DH, Hwang KY: Associations between laboratory parameters and outcome of paraquat poisoning. Toxicol Lett, 2000; 118: 53-59

12. Suzuki K, Takasu N, Arita S et al: A new method for predicting the outcome and survival period in paraquat poisoning. Hum Toxicol, 1989; 8: 33-38

13. Lee $\mathrm{Y}$, Lee $\mathrm{JH}$, Seong $\mathrm{AJ}$ et al: Arterial lactate as a predictor of mortality in emergency department patients with paraquat intoxication. Clin Toxicol, 2012; 50: 52-56

14. Choi JS, Kwak KA, Park MJ et al: Ratio of angiopoietin-2 to angiopoietin-1 predicts mortality in acute lung injury induced by paraquat. Med Sci Monit, 2013; 19: 28-33

15. Huang NC, Lin SL, Hung YM et al: Severity assessment in acute paraquat poisoning by analysis of APACHE II score. J Formos Med Assoc, 2003; 102: 782-87

16. Huang NC, Hung YM, Lin SL et al: Further evidence of the usefulness of Acute Physiology and Chronic Health Evaluation II scoring system in acute paraquat poisoning. Clin Toxicol, 2006; 44: 99-102

17. Weng $\mathrm{CH}$, Hu CC, Lin JL et al: Sequential organ failure assessment score can predict mortality in patients with paraquat intoxication. PLoS One, 2012; 7: e51743

18. Sawada Y, Yamamoto I, Hirokane T et al: Severity index of paraquat poisoning. Lancet, 1988; 1: 1333

19. Min YG, Ahn JH, Chan YC et al: Prediction of prognosis in acute paraquat poisoning using severity scoring system in emergency department. Clin Toxicol, 2011; 49: 840-45

20. Zhou CY, Kang X, Li CB et al: Pneumomediastinum predicts early mortality in acute paraquat poisoning. Clin Toxicol, 2015; 53: 551-56

21. Kang X, Hu DY, Li CB et al: The volume ratio of ground glass opacity in early lung CT predicts mortality in acute paraquat poisoning. PLoS One, 2015; 10: e121691

22. Pieper-Bigelow $C$, Strocchi A, Levitt MD: Where does serum amylase come from and where does it go? Gastroenterol Clin North Am, 1990; 19: 793-810

23. Warshaw AL, Lee $\mathrm{KH}$ : Characteristic alterations of serum isoenzymes of amylase in diseases of liver, pancreas, salivary gland, lung, and genitalia. J Surg Res, 1977; 22: 362-69

\section{Acknowledgements}

We thank the physicians and nurses of our institutions.

\section{Declaration of interest}

The authors report no conflicts of interest. The authors alone are responsible for the content and writing of the paper.

24. Jam I, Shoham M, Wolf RO, Mishkin S: Elevated serum amylase activity in the absence of clinical pancreatic or salivary gland disease: Possible role of acute hypoxemia. Am J Gastroenterol, 1978; 70: 480-88

25. Gil HW, Yang JO, Lee EY, Hong SY: The level and clinical significance of pancreatic enzymes in survivors of acute paraquat poisoning. Clin Toxicol, 2009; 47: 308-11

26. Li Y, Wang M, Gao Y et al: Abnormal pancreatic enzymes and their prognostic role after acute paraquat poisoning. Sci Rep, 2005; 5: 17299

27. Lin JL, Leu ML, Liu YC, Chen GH: A prospective clinical trial of pulse therapy with glucocorticoid and cyclophosphamide in moderate to severe paraquat-poisoned patients. Am J Respir Crit Care Med, 1999; 159: 357-60

28. Khwaja A: KDIGO clinical practice guidelines for acute kidney injury. Nephron Clin Pract, 2012; 120: c179-84

29. Sarr MG: 2012 revision of the Atlanta classification of acute pancreatitis. Pol Arch Med Wewn, 2013; 123: 118-24

30. Banks PA, Bollen TL, Dervenis C et al: Classification of acute pancreatitis 2012: Revision of the Atlanta classification and definitions by international consensus. Gut, 2013; 62: 102-11

31. Yang JO, Gil HW, Kang MS et al: Serum total antioxidant statuses of survivors and nonsurvivors after acute paraquat poisoning. Clin Toxicol, 2009; 47: 226-29

32. Seok S, Kim YH, Gil HW et al: The time between paraquat ingestion and a neg ative dithionite urine test in an independent risk factor for death and organ failure in acute paraquat intoxication. J Korean Med Sci, 2012; 27: 993-98

33. Houze $P$, Baud FJ, Mouy R et al: Toxicokinetics of paraquat in humans. Hum Exp Toxicol, 1990; 9: 5-12

34. Soontornniyomkij V, Bunyaratvej S: Fatal paraquat poisoning: a light microscopic study in eight autopsy cases. J Med Assoc Thai, 1992; 75(Suppl.1): 98-105

35. Gottlieb K, Sherman S, Pezzi J et al: Early recognition of post-ERCP pancreatitis by clinical assessment and serum pancreatic enzymes. Am J Gastroenterol, 1996; 91: 1553-57

36. Paajanen $\mathrm{H}$, Nuutinen $\mathrm{P}$, Harmoinen $\mathrm{A}$ et al: Hyperamylasemia after cardiopulmonary bypass: Pancreatic cellular injury or impaired renal excretion of amylase? Surgery, 1998; 123: 504-10

37. Frulloni L, Patrizi F, Bernardoni L, Cavallini G: Pancreatic hyperenzymemia clinical significance and diagnostic approach. JOP, 2005; 6: 536-51

38. Collen MJ, Ansher AF, Chapman AB et al: Serum amylase in patients with renal insufficiency and renal failure. Am J Gastroenterol, 1990; 85: 1377-80

39. Junge W, Malyusz M, Ehrens HJ: The role of the kidney in the elimination of pancreatic lipase and amylase from blood. J Clin Chem Clin Biochem 1985; 23: 387-92

40. Vaziri ND, Ness RL, Fairshter RD et al: Nephrotoxicity of paraquat in man Arch Intern Med, 1979; 139: 172-74

41. Kim SJ, Gil HW, Yang JO et al: The clinical features of acute kidney injury in patients with acute paraquat intoxication. Nephrol Dial Transplant, 2009; 24: 1226-32

42. Cote GA, Gottstein JH, Daud A, Blei AT: The role of etiology in the hyperamylasemia of acute liver failure. Am J Gastroenterol, 2009; 104: 592-97

43. Ede RJ, Moore KP, Marshall WJ, Williams R: Frequency of pancreatitis in fulminant hepatic failure using isoenzyme markers. Gut, 1988; 29: 778-81

44. Srinivasan A, Venkataraman S, Hansdak SG et al: Hyperglycaemia as an indicator of concurrent acute pancreatitis in fulminant hepatic failure associated with hepatitis B infection. Singapore Med J, 2005; 46: 236-37

45. Lippi G, Valentino M, Cervellin G: Laboratory diagnosis of acute pancreatitis: In search of the Holy Grail. Crit Rev Clin Lab Sci, 2012; 49: 18-31

46. Skrha J, Stepan J: Clinical significance of amylase isoenzyme determination. Acta Univ Carol Med Monogr, 1987; 120: 1-81 